\section{Zentrale Störungen mit exzessiver}

\section{Tagesschläfrigkeit}

Helga Peter ${ }^{1}$ und Thomas Penzel ${ }^{2}$

${ }^{1}$ Marburg, Deutschland

${ }^{2}$ Interdisziplinäres Schlafmedizinisches Zentrum, Charité Universitätsmedizin Berlin, Berlin, Deutschland

\section{Synonyme}

Zentralnervöse Störungen mit schwergradiger Tagesschläfrigkeit

\section{Englischer Begriff}

central disorders of hypersomnolence

\section{Definition}

In der $\downarrow$ „ICSD-3“ (2014) ersetzt der Begriff die in der ICSD-2 gewählte Bezeichnung Hypersomnien zentralnervö- sen Ursprungs (hypersomnias of central origin). Die Diagnosegruppe umfasst Erkrankungen, die zentralnervösen Ursprungs sind und mit der Hauptbeschwerde der exzessiven Tagesschläfrigkeit einhergehen. Dazu gehören

- „Narkolepsie“

- „Kleine-Levin-Syndrom“

- „Idiopathische Hypersomnie“

- „Schlafmangelsyndrom“

\section{Ferner}

- Hypersomnie durch körperliche Erkrankung

- Hypersomnie durch Medikamente oder Substanzen

- Hypersomnie assoziiert mit psychischer Erkrankung

- Langschläfer (klassifiziert als Isoliertes Symptom oder Normvariante)

Siehe auch

- \Medikamentennebenwirkungen“

- „Symptomatische Schlafstörungen“ 